\title{
UNA APROXIMACIÓN TEÓRICA A LA RELACIÓN DE LA EMPRESA TURÍSTICA CON LA ÉTICA Y LA RESPONSABILIDAD SOCIAL
}

\author{
Alicia Ledesma \\ Universidad Nacional de Rosario
}

\begin{abstract}
Resumen: Dadas las condiciones imperantes de sostenibilidad global es conveniente encontrar otros paradigmas que permitan el estudio del impacto y la generación de negocios turísticos inclusivos y equitativos. En otras palabras, se debe pensar a los negocios turísticos desde una concepción basada en valores éticos y sociales, que desarrollen vínculos interdisciplinarios y analicen las cuestiones sociales, económicas, históricas, culturales y naturales (medioambientales) presentes en la consolidación de todo desarrollo turístico verdadero. Mediante una aproximación teórica, este trabajo intenta traer una reflexión sobre la relación que existe entre la empresa turística, la sociedad la ética y la responsabilidad social.
\end{abstract}

Palabras Claves: empresa turística, ética, responsabilidad social, stakeholders.

Resumo: Unha aproximación teórica á relación da empresa turística coa ética e a responsabilidade social.

Dadas as condicións imperantes da sustentabilidade global é conveniente encontrar outros paradigmas que permitan tanto o estudo do impacto da xeración de negocios turísticos como a análise da creación de negocios turísticos inclusivos e equitativos. Noutras palabras, débese pensar os devanditos negocios desde unha concepción baseada en valores éticos e sociais, que desenvolvan vínculos interdisciplinarios e analicen as cuestións sociais, económicas, históricas, culturais e naturais (ambientais) que deben estar presentes na consolidación de calquera desenvolvemento turístico verdadeiro. Mediante unha aproximación teórica, este traballo intenta reflexionar sobre a relación que existe entre a empresa turística, a sociedade, a ética e a responsabilidade social.

Palabras clave: empresa turística, ética, responsabilidade social, partes interesadas (stakeholders).

\footnotetext{
Abstract: $A$ theoretical approach to the relationship of tourism companies with ethics and social responsibility.

Given the prevailing global sustainability conditions, it is important to find other paradigms which allow us to study the impact and generation of inclusive and equitable tourism businesses. In other words, we need to think about tourism businesses starting from a concept based on ethical and social values, which develops interdisciplinary links and analyses the social, economic, historical, cultural and natural (environmental) issues present in the consolidation of every true tourism development. By means of a theoretical approach, this work aims
} 
to reflect on the relationship which exists between tourism companies, society, ethics and social responsibility.

Key words: tourism company, ethics, social responsibility, stakeholders

\section{INTRODUCCIÓN}

A lo largo de la última década se ha oído alzar la voz a sociólogos y filósofos alertando sobre la necesidad de un manejo ético de las relaciones interpersonales, y por ende de los negocios y la política. Estas voces ya no portaban el rótulo de ninguna ideología, sino simplemente - y nada menos - una clara lectura e interpretación de la realidad en la que estábamos inmersos. Han pasado ya algunos años desde esas primeras voces, y empieza a cobrar entidad la importancia de esas palabras, de lo difícil que a veces resulta implementar cambios de conducta pero de lo imprescindible que se hace intentarlo. En la medida que todos los estamentos sociales lo entiendan, su aplicación será más sencilla. El negocio turístico no es ajeno a esta realidad.

El empresario turístico de este siglo XXI tiene ante sí un gran desafío: desarrollar negocios en un todo de acuerdo con el concepto de Desarrollo Sostenible, lo cual implica su compromiso con un desempeño ético y responsable. Algunas organizaciones ya han entendido la responsabilidad que les compete y la han hecho parte de su "misión" lo que a la vez le ha permitido mejorar considerablemente su rentabilidad,. como así lo expresa U. Gary Charlwood -Fundador, Presidente y CEO de Uniglobe trade- (WTTC, 2002: 1), “... A la compañía la guía la filosofía de contribuir con la sociedad y a la larga, no sólo es lo correcto de hacer, sino que es bueno para el negocio ya que crea una cultura organizacional colaborativa que es la llave para mantener motivados a los empleados...".

Ya los griegos, al fomentar la realización de los juegos panhelénicos (deporte, teatro, música) de modo de contribuir no sólo al desarrollo de la cultura, sino también a la pacificación e integración de los pueblos, sabían que el intercambio de experiencias entre los pueblos era beneficioso, tanto para el espíritu como para el cuerpo de sus habitantes y ese mismo espíritu es el que llevó a Pierre de Coubertain en 1896 a revivir los juegos olímpicos de la antigüedad. Hoy, bajo ese mismo ideario se dice que el Turismo contribuye al diálogo entre las naciones y las distintas culturas.

La práctica del turismo permite tomar distancia de la vida diaria, del trabajo, de las obligaciones a las que se está necesariamente atado. Esta distancia permite al hombre descubrirse él mismo y a los demás, tanto como individuos y como colectividad. Es así como se encuentra con "el otro", está obligado a compartir con "el otro", a convivir con "el otro". De esta forma el turista capta otras energías, otras imágenes, aprende nuevas palabras, conoce nuevas personas, se interesa por sus costumbres, sus condiciones de vida, su religión, sus problemas, admite la diversidad de un mundo al que le es imposible abarcar completamente. Este esfuerzo, sin lugar a dudas, lo hará aprender a apreciar lo que lo rodea y a darse cuenta sobre la necesidad de protegerlo. 
Como se mencionó, el conocimiento mutuo entre los individuos y los encuentros e intercambios culturales facilitan las relaciones de paz entre los pueblos, siendo el turismo uno de los actores en la estimulación de esas distintas formas de participación entre las culturas. Ahora bien, para lograr que la actividad turística esté siempre al servicio de la persona y la comunidad, es indispensable que todas las estructuras del país y sus actores sociales trabajen en conjunto, lo cual se logra garantizando a los habitantes de las localidades turísticas una participación en la planificación de la actividad, precisando muy bien los límites económicos, ecológicos y sociales. De esta manera una planificación y gestión responsable de la actividad convierte al turista en un colaborador solidario, exigente consigo mismo, con sus pares y con los organizadores del viaje. Es entonces, responsabilidad del turista (consumidor) la selección de los servicios ofrecidos por el mercado y necesarios para un desempeño eficiente, es decir que esta tarea debe realizarse individualmente y con la ayuda de habilidades de consumo y patrones de decisión adquiridos individualmente (Bauman, 2007).

En esta sociedad globalizada, el consumismo se ha convertido en el eje de la actividad turística. En un mercado ansioso de novedades, se venden sueños, fantasías, esperanzas e imaginarios sociales. Este impulso consumista transformó en bienes de consumo al medio ambiente, es decir a los recursos naturales y a los culturales, por lo que se debe tratar de evitar caer en el empobrecimiento progresivo de estos recursos como respuesta a las demandas de turistas ávidos de banalidades. Parafraseando a Marc Augé (2007: 59 y 70) se puede decir que los paisajes se han convertido en un producto más y se amontonan, unos sobre otros en los catálogos y en las pantallas de las agencias de viaje, así entonces tenemos que el turista moderno, es un simple consumidor que se considera viajero. Dado que es preciso hacer todo lo posible para que el turismo no llegue a ser, en ningún caso, una forma moderna de explotación, sino que sea la ocasión de un útil intercambio de experiencias y de diálogo fructífero entre los pueblos, es que la Organización Mundial del Turismo, sanciona el Código Ético Mundial para el Turismo, con la intención que sea el marco de referencia para que las diferentes empresas del sector, puedan desarrollarse reduciendo al mínimo los efectos negativos de esta actividad sobre el medio ambiente y el patrimonio cultural, al tiempo que aprovechen al máximo sus beneficios en la promoción del desarrollo sostenible y el alivio de la pobreza y el entendimiento entre las naciones. Así entonces, la propuesta de este código debería ser la base de una profunda discusión entre los actores locales pero al no ser éste el eje de estas líneas sólo se hará mención a que "la normativa que contiene no debería tener un carácter apodíctico, sino ser la plataforma conceptual para una amplia y profunda discusión en el seno de las comunidades involucradas" (Winger, 2008: 14).

Retomando lo planteado anteriormente, "la ética ha sido el lado olvidado en los procesos de racionalización económica moderna y es hora de hacer la prueba de activar las virtualidades de la economía capitalista (Cortina, 2000: 66-67) para lo cual es necesario como postulaba Aristóteles que para "ser justo...es indispensable adquirir el hábito de tomar decisiones justas, igual que para ser prudente, fuerte o magnánimo”. (Cortina, 2003: 19) 


\section{LA EMPRESA TURÍSTICA Y LA ÉTICA}

A raíz de lo expuesto se puede pensar que el negocio turístico está atravesado por el "utilitarismo" doctrina ética que, a pesar de sus limitaciones, puede ser empleada en este contexto. Lo expuesto a lo largo de los párrafos anteriores ejemplifica las palabras que utiliza Esperanza Guisán (1992) para definir al utilitarismo al decir que sus principios afirman que la felicidad es el valor más importante a nivel individual, que la utilidad general, el bien común y el bienestar colectivo es la meta deseable en el quehacer de los gobiernos. Pero, ¿se puede utilizar este abordaje al hablar de las empresas, o no sería más adecuado considerar una perspectiva que se centre en la ética personal? Esta posición pone en evidencia que aunque "los integrantes de la organización tengan un comportamiento ético no quiere decir que la empresa actúe del mismo modo". Aquí se plantea entonces, la dificultad de reconocer una conducta ética única, ya que ésta va a depender de la teoría utilizada como patrón de referencia. De este modo, ética y realidad organizacional serían dos contenidos distintos a ser considerados en una reflexión teórica sobre la toma de decisiones.

Un abordaje completamente distinto es el que encuentran en la economía en general, y en las ciencias sociales en particular, las bases de la ética. Desde este enfoque ya Adam Smith, en su obra "Riqueza de las Naciones" en el siglo XVII expone la compatibilidad entre ética y beneficio al afirmar que el beneficio no es un agregado indebido sino un vector de renta y de promoción social. A su vez, Amartya Sen al analizar la obra de Smith sostiene que “... analiza toda una variedad de valores implícitos en las relaciones económicas, sociales y políticas que muchos de sus continuadores olvidaron representar en sus modelos..." (Gómez Fulao, 2003: 165). Desde este punto de vista entonces, se debe considerar a la ética como una variable del proceso económico, perceptible concretamente, en la práctica del management en general y en el de las empresas turísticas en particular.

En este sentido y parafraseando a Amartya Sen (Cortina, 2003: 43) se puede decir que el éxito de las empresas depende de las conductas, preocupaciones y valores de un grupo humano más amplio que el de los empresarios -lo cual en el negocio turístico es de una exactitud asombrosa. Por tanto debe considerarse el alcance de la ética empresarial de un modo lo suficientemente amplio e inclusivo, siendo su papel primordial hacer posible la cooperación y la interacción empresarial. Utilizando una de las clasificaciones propuestas por la filosofía, la ética puede clasificarse en: ética axiológica -se ocupa de analizar el sentido y la justificación de los conceptos éticos-, ética dialógica -saca a la luz las condiciones que hacen de un diálogo un discurso racional, empeñado en hallar la verdad de las proposiciones teóricas y la corrección de las normas prácticas- y ética aplicada -la puesta en acción de los valores analizados en las ética axiológica y dialógicasAsí entonces, es desde la ética aplicada que se puede hablar de la ética empresarial o de la empresa.

En función de la naturaleza de la actividad turística y el devenir de los negocios hoy, comenzar a pensar los negocios turísticos y por ende la actuación de las organiza- 
ciones del sector desde la perspectiva de la ética se ha vuelto imprescindible. Para ello es necesario tomar a la ética de la empresa o ética empresarial (aplicada) en un marco referencial que trabaje en tres dimensiones: la ética de la cultura; la ética de la dignidad humana y la ética de la responsabilidad.

En este punto no se puede dejar de considerar que para no caer en un simple reduccionismo, la ética debe interactuar con los problemas de la organización y del ejercicio comercial sin perder la perspectiva ética orientadora del desarrollo en el tratamiento de estos problemas. Aún a riesgo de ser reiterativos es importante remarcar que el éxito de la empresa depende de las acciones de los individuos o grupos de individuos cuyos intereses están en juego en la gestión de dicha empresa. Como se planteara en el párrafo anterior, es necesario tomar un marco referencial que trabaje las tres dimensiones: la ética de la dignidad humana (trabaja sobre la dignidad del ser humano), la ética de la cultura (trabaja sobre los sujetos para lograr prácticas compartidas en la organización) y la ética de la responsabilidad (trabaja sobre las consecuencias de las acciones). Por el contrario, si se toma cada una de estas dimensiones en forma aislada se caería en un reduccionismo que la ética de la empresa no se merece. En este caso, la dimensión de la responsabilidad se reduciría a ignorar los criterios y finalidades que la hacen inteligible y los principios que la enmarcan como la atención a los sujetos que actúan. Así entonces, la de la dignidad humana se reduciría a la enumeración de principios abstractos insensibles al contexto, indiferentes a las consecuencias e ignorantes de los sujetos concretos y la de la cultura a una identidad cultural cerrada en sí misma, que no resiste las críticas y no considera sus responsabilidades.

A través del siguiente cuadro se visualizan sus caracterizaciones más importantes:

Cuadro 1: Marco Referencial de la ética organizacional

\begin{tabular}{lll} 
Dimensiones & Trabaja sobre & Se materializa en \\
\hline De la dignidad Humana & La dignidad del Ser Humano & $\begin{array}{l}\text { Códigos, misiones y } \\
\text { principios empresariales }\end{array}$ \\
\hline De la Cultura & Los sujetos y la cultura & Ethos Organizacional \\
\hline De la Responsabilidad & Las consecuencias de las acciones & $\begin{array}{l}\text { Responsabilidad Social } \\
\text { Empresaria }\end{array}$ \\
\hline
\end{tabular}

Fuente: Elaboración propia

Para Lozano (1999) las organizaciones no trabajan directamente con el principio de la dignidad humana, sino que en sus procesos de construcción de criterios, valores y metas empresariales, utilizan el "reconocimiento del otro" por lo que se trata entonces, de un momento interno, no necesariamente visible. De esta manera, la construcción del ethos organizacional dependerá del actuar de cada uno de los individuos en su propio contexto organizacional, del ejemplo de los directivos y de la cohesión que exista entre los actos 
y los valores enarbolados por la organización. Es así cómo, en la formación de este ethos organizacional, la persona y la cultura organizacional son sus elementos fundamentales.

Dado el carácter particular de las empresas del sector turístico ${ }^{1}$, ya sea por la compleja problemática social y económica a la que se encuentra sometida como por la gran cantidad de servicios que conforman su producto, es fundamental que éstas cumplan con la condición de "abiertas", es decir que la empresa debe empezar por sí misma, debe hacer de sus propios procedimientos internos un ejemplo para, desde adentro, avanzar a la comunidad. De lo contrario, se reduce a tener normas y prohibiciones que nacen y mueren en la misma empresa. La empresa que sea capaz de cumplir con esta condición de "abierta" se encontrará con una ética que actúa como columna vertebral de todo su proceso organizacional y no con una estrategia que tome en cuenta algunos comportamientos éticos o responsables por una exigencia del ambiente externo. Esto es así porque la ética, vista desde dentro hacia fuera, ilumina cada una de las dimensiones organizacionales y las convierte en estrategias para formular buenas prácticas organizacionales. (Ledesma, 2007)

\subsection{LOS STAKEHOLDERS}

Desde una perspectiva económica, al turismo se lo ha considerado un motor para el desarrollo de los países, dependiendo éste de factores tales como las condiciones de mercado, la tecnología, la educación y hasta los sistemas tributarios entre otros. Sobre lo que no hay lugar a dudas es que la oferta turística es uno de ellos desde el momento que se la considera como el conjunto de diversos bienes y servicios que relacionados entre sí logran la satisfacción de las necesidades de los turistas.

En este contexto, las empresas turísticas adquieren cada vez mayor importancia económica no sólo por la cantidad de empleos que generan sino porque son el soporte de las corrientes turísticas que llegan a un destino (Vargas: 2007) y a su vez, su actividad se desarrolla en escenarios que son de la sociedad toda: bienes históricos, parajes naturales, monumentos y lugares que se identifican como patrimonio de la humanidad, Sin embargo, y “... dada su participación en los procesos sociales de las comunidades y su relación con la naturaleza; se hace necesario identificar bajo qué condiciones se producen y prestan estos servicios turísticos ..." (Vargas, 2007: 10). En otras palabras, cuál es la responsabilidad de estas empresas en lo que al medio ambiente y a la sociedad se refiere. Es en este momento entonces que se puede pensar esta relación desde la Teoría de los

1 En lo que se refiere a la empresa turística y siguiendo a Figuerola Palomo ( $\mathrm{s} / \mathrm{f}$ ) podemos sostener que una empresa puede ser considerada turística, ya sea que provenga del sector primario, industrial o terciario si cuando su producción esté dirigida al consumo turístico puesto que el concepto de turismo nunca puede estar ligado o relacionado con una actividad sectorial en tanto y en cuanto la valoración del turismo no se identifica directamente con ningún proceso productivo concreto sino que por el contrario, la naturaleza turística procede del consumidor que absorbe los bienes y servicios generados. De esta manera su gestión y organización se ve afectada por ciertos elementos tipificadores (Casanova Rocha et al., 2000) entre los que encontramos a la estacionalidad, a la intangibilidad e inmaterialidad de su producción, a la necesidad de una elevada inversión, a la inflexibilidad e inmovilidad de los servicios prestados, a la localización próxima a los recursos turísticos, a la necesidad de contar con recursos humanos capacitados, a los cambios en los gustos y expectativas de los consumidores y a la importancia que tienen los procesos de intermediación en el negocio. 
Stakeholders, entendiendo por estos últimos a todos los actores que de una u otra manera se ven afectados, positiva o negativamente, por el accionar de la empresa en cuestión.

Esta teoría, desarrollada por Freeman en los años ochenta, sostiene que la empresa en su dirección y gobierno es responsable no sólo de los accionistas sino también de otros individuos y/o grupos que tienen intereses en las acciones y decisiones de dicha organización (Lozano, 1999). Dicho de esta manera, bien puede pensarse que es algo muy simplista, pero realmente no lo es. En lo que respecta a la gobernabilidad de las empresas, esta teoría se sustenta en la búsqueda, por parte de la dirección, de un equilibrio entre las exigencias legítimas de sus distintos grupos de intereses de la empresa con los objetivos de la organización. De esa manera, nos encontramos con una concepción de la responsabilidad de las empresas donde tanto los directivos como la misma empresa son y deberían ser vistos como agentes morales que representan los intereses o exigencias de una gran variedad de grupos. Por lo tanto, se hace necesario establecer políticas, estrategias, procesos y hasta una cultura empresarial propia en sintonía con el concepto de empresa como stakeholder lo que le permitirá generar procedimientos y respuestas ante esa responsabilidad. En este sentido, la teoría de los stakeholders sostiene que es imposible separar los problemas económico-empresariales de los problemas o cuestionamientos éticos, lo cual hace que al momento de preguntarse cuál debería ser el modo de actuar de la empresa ante tal situación, se deba reflexionar sobre los valores morales de la organización para poder elegir entre las diferentes políticas, estrategias y acciones aquellas que son más acordes económica y éticamente- con el concepto o la misión de la empresa tanto en su corto, medio como largo plazo. En palabras de Harrison y Freeman (Harrison et al., 1999: 483484) “....seguramente los efectos económicos son también sociales, y seguramente los efectos sociales son también económicos. Dividir el mundo en económico y social, en definitiva, es bastante arbitrario. Desde luego, una de las ideas originales detrás del enfoque de gestión de Stakeholder era tratar de encontrar un camino para integrar lo económico y lo social". No obstante, uno de los problemas que se presenta entonces, es que la teoría no ofrece un punto de vista moral o criterio ético desde el cual se pueda diferenciar entre las exigencias que son legítimas y las que no lo son y, en este sentido, se presenta un problema práctico al tratar de responder a la responsabilidad que hace referencia la Teoría de Stakeholder, 'ser responsable de las exigencias de sus stakeholders'.

Entre otras cuestiones, este problema dio origen a las numerosas críticas que recibió esta teoría, una de las cuales se basa en que la responsabilidad de las empresas y de su dirección sólo se deriva de la naturaleza de los contratos explícitos y concretos y nunca de las relaciones -implícitas o explícitas- con cada uno de los Stakeholders, en razón de la amplitud del concepto de responsabilidad que maneja esta teoría.

La respuesta de Freeman a estas críticas no se hace esperar y redefine su concepto de empresa ${ }^{2}$ como 'un nexo de contratos explícitos e implícitos', a los que denomina

2 En 1988 Freeman define a la empresa como una colección de acuerdos voluntarios entre adultos que consienten voluntariamente su adhesión al objetivo y fines de la corporación $\mathrm{y}$, a entrar en acuerdos cooperativos con otros individuos o grupos. 
"Stakeholders como partes contratantes" (Stakeholder-as-contrator), pero basándose en la intersubjetividad de la teoría de la Justicia de Rawls y descartando el monologismo kantiano anterior, así entonces Freeman reelabora la teoría de los Stakeholders basada en la idea del contrato social. En palabras del propio Freeman ... "El marco de pensamiento kantiano, sin embargo, obliga a imponer un mandato sobre este que proceso que no admite el pluralismo en el que nosotros estamos, de modo obvio inmersos. (...) Si nosotros comenzamos con el punto de vista de que podemos comprender la actividad de creación de valor como un proceso contractual entre esas partes afectadas, y si en aras de la simplicidad inicialmente designamos a esas partes como financistas, clientes, suministradores, empleados, y comunidades, entonces podemos construir un núcleo normativo que refleje las nociones liberales de autonomía, solidaridad, y las nociones liberales de autonomía, solidaridad, y racionabilidad tal y como son articuladas por John Rawls, Richard Rorty y otros" (Freeman, 1994 en Gonzáles, 2003: 7). Así, esta teoría rediseñada se basa en la idea liberal de justicia, entendida como la igualdad entre las partes contratantes, por lo cual se formula un contrato justo del cual se deriva la responsabilidad que la organización posee en relación con sus stakeholders. Llegando a afirmar que un contrato es justo si todas las partes intervinientes en el contrato pudieran estar de acuerdo con todos los términos del contrato bajo ignorancia de sus actuales intereses, lo que, a los ojos de Freeeman, únicamente puede hacer considerar un contrato como justo o como 'una apuesta justa'.

\section{LA EMPRESA TURISTICA Y LA RESPONSABILIDAD SOCIAL}

Figuerola (1991:15) define a las empresas turísticas bajo tres concepciones: una como la unidad económica que ejecuta el proceso de producción de bienes y servicios; otra, como el conjunto de factores de producción que ordenados en relación con principios de carácter social y tecnológico, persigue objetivos de tipo económico; y una tercera como la unidad económica, jurídica y social de explotación que formada por los factores productivos necesarios y bajo una dirección estratégica, persigue la obtención de valores agregados mediante la producción de bienes y servicios. Al hacer una revisión de la literatura se encuentra, sin importar desde qué concepción se tome, que hay quienes afirman que la empresa tiene responsabilidades definidas, ser rentable, competitiva y productiva, por lo que no se le deben atribuir responsabilidades adicionales (González, 2001: 3), pero también hay quienes opinan acerca del compromiso que debe existir por parte de la empresa en aspectos de corte legal, filantrópico, ético y económico. Friedman, puntualizó en sus publicaciones el papel de la empresa bajo el enfoque de negocios en donde considera que la responsabilidad social de las empresas es utilizar sus recursos y dedicarlos a aquellas actividades que aumenten los beneficios de los accionistas, siendo a partir de éstos que se pueden generar las obligaciones morales, sin sacrificar la rentabilidad de la empresa. Sin embargo, es necesario recordar el pensamiento de Kohlberg que sostiene que las decisiones son justas cuando se las toma poniéndose en el lugar del otro, lo cual excluye tener por justo lo que sólo satisface las necesidades de un 
grupo (Cortina, 2000: 16). Así entonces se puede decir que las obligaciones de la empresa no están limitadas a los accionistas, sino que comprenden a un grupo mayor, los stakeholders, como ya se explicitara, todos los beneficiados o afectados por la empresa a partir de sus operaciones. Desde esta óptica se requiere una actitud de respeto por parte los actores involucrados tanto receptores como visitantes, en donde se transforme la objetivación de la naturaleza y su explotación como recurso escaso, limitado y con valor económico de modo de lograr un disfrute del turismo como un espacio común de encuentro con un paisaje, modificado o socialmente construido (Rozo en Meyer, 2002: 125-140). No se debe olvidar que para un desarrollo sustentable del turismo, las poblaciones locales deben estar involucradas ${ }^{3}$, por lo que se deberá realizar un proceso participativo que garantice el conocimiento y revalorización de los valores culturales, con acciones que le permitan a la comunidad asimilar y comprender esta actividad de modo que pase a formar parte de sus actividades económicas y sociales.

Siguiendo el pensamiento de Aranguren (Cortina, 2000: 9) “...existe la necesidad de vivir el concepto de empresa como quehacer moral, el quehacer por excelencia de nuestro tiempo, cuyos objetivos son no sólo la producción y los beneficios que de ella, de los bienes y servicios resultan, sino el desarrollo humano y el bien moral...". Es en este punto donde se abre el debate acerca del servicio que las empresas turísticas deben prestar a una sociedad que a su vez constituye su mercado y de cómo esta sociedad influye en su comportamiento, exigiéndoles ciertas conductas y sancionándolas por su incumplimiento. A medida que aumenta la preocupación por la satisfacción de las necesidades de los turistas y de la sustentabilidad de los destinos, mayor es la presión pública a la que se ven sometidas las empresas turísticas para asumir esa responsabilidad (Vargas, 2007).

Los turistas se ven impactados y atraídos por los recursos (naturales y culturales) de un cierto destino dado que están siempre buscando experiencias y conocimientos diferentes. Hoy en día, "son más numerosos los turistas con sensibilidad ambiental que buscan destinos bien planificados y menos contaminados, y que dejan a un lado los mal diseñados con problemas ambientales o sociales" (OMT, 1999: 21). La exigencia para el turismo hoy en día, es lograr un equilibrio entre la satisfacción de necesidades humanas y la actividad económica.

Pero a pesar de lo dicho, este sector se mueve con tranquilidad dado que cuenta con una mejor imagen pública ambiental y social. De esa manera se explica por qué la actitud de estas empresas hacia la responsabilidad social está en función de las presiones del entorno en el que se desenvuelven. Es en este momento entonces cuando los stakeholders (turistas, trabajadores, asociaciones, aseguradoras, etc.) adquieren un papel significativo en las decisiones que la empresa toma hacia la implementación de una estrategia de responsabilidad social. Hoy en día las empresas turísticas reconocen la necesidad de tener una correcta gestión, ya que se enfrentan no sólo al reto de ser

3 El turismo es una herramienta sumamente efectiva de desarrollo regional, en la medida que sea entendido como un medio y no como el fin último de las actividades económicas de las comunidades (Rozo, 2002: 133). 
competitivas, sino también al de considerar los factores a favor de la sustentabilidad de los destinos.

La aceptación de la responsabilidad que tienen las empresas con su entorno forma parte de una evolución imparable, en la que a medida que las unidades empresariales han ido incrementando su tamaño y su peso relativo en la economía, se han visto obligadas a asumir responsabilidades de carácter social que van más allá de las puramente económicas, tiene que ver con sus trabajadores, con el ambiente, con sus clientes, con la comunidad local, etc. (Llull, 2003: 33). De esta manera, si entre los objetivos de toda empresa está el de lograr beneficios económicos, valdría la pena reflexionar en el camino que los empresarios siguen para obtenerlo. Los procesos de operación del servicio turístico son complejos, entre otras cosas porque involucran a muchas personas y acciones, por lo cual las consecuencias de su participación pueden no ser del todo responsables. Cabe aquí entonces reflexionar sobre el pensamiento de Adela Cortina (2000) cuando sostiene que para algunas personas hablar de negocios compatibilizando términos tales como "ética" y "empresa" puede llegar a ser una tarea titánica y hasta imposible puesto que pareciera que vivir ética o moralmente sólo se logra si se evita cualquier contacto con el mundo de los negocios. Ahora bien, ¿es esto así? No será más bien una forma inadecuada de entender tanto a la ética como a la empresa?

Como ya se planteara, en el sector turístico la responsabilidad social es de especial importancia, ya que por una parte, la empresa impacta en una gran cantidad de factores en los destinos donde se ubica, pero también por la otra, dado el sistema en que se encuentra inmersa, porque los servicios turísticos dependen mucho más de su buena reputación que empresas de cualquier otro sector. Por tal motivo, es de esperar que las empresas turísticas cumplan con una serie de condiciones que garanticen primeramente el control de sus actuaciones y después, que aumenten su credibilidad ante la opinión pública y ofrezcan confianza y seguridad a los turistas (Vargas, 2007).

En este contexto en el que se ha planteado la responsabilidad de las empresas turísticas y tal como se desprende del texto, no puede desconocerse a la ética ya que ésta se torna una exigencia externa a causa de las demandas sociales. Ahora bien, cuando la sociedad le demanda a una empresa que actúe con rectitud en su relación con el ambiente, la ética se convierte en un elemento fundamental de la estrategia de aquella, que busca adaptar su sistema formal a esa necesidad de la sociedad. En ese sentido entonces, su accionar social es lo que se ha dado en llamar responsabilidad social de la empresa, constituyendo un elemento importante de la aplicación de la ética empresarial.

Así entonces, se plantea fundamental el comprender que no por hablar de una conducta y compromiso ético, se está hablando de conductas no rentables, sino por el contrario se está hablando de implementar conductas que permitan seguir teniendo rentabilidad a lo largo del tiempo y aún a riesgo de ser reiterativo, esto se logra cuando todos los actores involucrados en el negocio turístico entiendan a la ética como columna vertebral de todo su proceso organizacional y no como una estrategia que tome en cuenta algunos comportamientos éticos o responsables por una exigencia del ambiente externo. 


\section{REFLEXIONES FINALES}

Se hace necesario entonces, reflexionar sobre el fin último de la empresa y a partir de allí sobre aquellas estrategias de crecimiento de las empresas turísticas que les permitan sostenerse por un largo periodo en el mercado respondiendo responsablemente a todos los que participan con ella y que no sólo tomen en cuenta a la responsabilidad social como un valor independiente que les permita mejorar su imagen social (Vargas, 2007).

En función de lo expuesto a lo largo del escrito y al tratar de reflexionar sobre este tema se nos presentan algunas cuestiones cuyas respuestas nos permitirán ahondar en la comprensión de la relación empresa turística -sociedad- ética- responsabilidad. Interrogantes tales como ¿qué factores intervienen en la determinación del óptimo económico, social y ecológico de una empresa turística?; ¿es posible aplicar un modelo de gestión sustentable bajo el paradigma de la economía clásica?; ¿cuál es el modelo de empresa turística que permite aplicar las ideas derivadas de la rediseñada teoría de los stakeholders?; ¿por qué la responsabilidad de la empresa hacia la sociedad debería ser incluida en los fines mismos (misión) de la gestión empresaria?; ¿lograr que la organización sea ética significa que no será rentable?, ¿en qué ámbitos debe trabajar la empresa turística para hacer efectiva la responsabilidad que le compete en la sociedad? nos permitirán abordar no sólo el rol de los stakeholders ante el concepto de responsabilidad social, sino determinar el tipo de gestión y de directivo que las empresas turísticas de este siglo XXI necesitan.

Indudablemente lo que hoy se necesita es el desarrollo de negocios turísticos inclusivos y equitativos -dentro de los parámetros de sostenibilidad global- para lo cual se está ante la necesidad fundada de adoptar otros paradigmas para el estudio del impacto y la generación de estos negocios turísticos. En otras palabras, se debe pensar en este tipo de negocios desde una concepción basada en estrategias fundamentadas en valores éticos y sociales, desarrollando vínculos interdisciplinarios, analizando cuestiones sociales, económicas, históricas, culturales y naturales (medioambientales) presentes en la consolidación de todo desarrollo verdadero. Se hace necesario entonces, reflexionar sobre el tipo de directivo que se necesita y sea capaz de llevar adelante aquellas estrategias de crecimiento de las empresas turísticas que les permitan sostenerse por un largo periodo en el mercado respondiendo responsablemente a todos los que participan con ella y que no sólo tomen en cuenta a la responsabilidad social como un valor independiente que les permita mejorar su imagen social.

\section{BIBLIOGRAFÍA}

Augé, M. (2007): Por una antropología de la movilidad, Barcelona, Gedisa.

Bauman, Z. (2007): Vida de Consumo, Buenos Aires, FCE.

Casanova Rocha, C., García del Junco, J., Caro Gonzáles, F. (2000): Organización y gestión de empresas turísticas, Madrid, Ed. Pirámide. 
Cortina, A. (2000): Ética de la Empresa, Madrid, Ed. Trotta.

Cortina, A (2003): “Construir confianza, Madrid, Ed. Trotta.

Ferrater Mora, J. (1986) Diccionario de Filosofia, Madrid, Alianza Editorial, pp $1.057 / 63$.

Figuerola, M. (1991): Elementos para el estudio de la economía de la empresa turistica, España, Síntesis, p. 15.

Figuerola Palomo, M. (s/f): Economía para la gestión de las empresas turísticas (Organización y financiación), Madrid, Ed. Centro de Estudios Ramón Areces SA, pp 46-50.

Gómez Fulao, J.C. (2005): Tiempos de economía, épocas de inequidad, Buenos Aires, Ed. Cooperativas.

González Esteban, E. (2000): “Consecuencialismo y deontologismo para una Ética Empresarial: Una mirada desde la Teoría de Stakeholders" trabajo presentada en el $2^{\circ}$ ISBEE World Congress Business, Economics and ethics Sao Paulo, Brazil 19 al 23 de Julio de 2000.

González Marroquín, G. (2001). Responsabilidad social de las empresas: alcance y cuestionamiento sobre el tema, ponencia presentada en el $5^{\circ}$ Congreso nacional e internacional realizado por la ADOARH en Revista IONTERFORUM, p. 3, Texas, USA.

Guisán, E. (1992): "Utilitarismo” en Camps, V.; Guaraglia, O. y Salmerón, F. (edit.) Concepciones de la Ética, Madrid, Editorial Trotta. Guaraglia.

Heler, M. (2002): “Cuestiones ético - profesionales"- Programa de Doctorado en Administración - Facultad de Ciencias Económicas y Estadísticas. Universidad Nacional de Rosario.

Hernández Sampieri, R; Fernández Collado, C.; Baptista Lucio, P. (2004): Metodología de la Investigación, México, Mc Graw Hill

Ledesma, A. (2007): La relación ética - estrategia en el negocio turístico ponencia presentada en las $\mathrm{VIII}^{\mathrm{o}}$ Jornadas Nacionales y II $^{\mathrm{o}}$ Simposio Internacional de Investigación - Acción en Turismo organizadas por CONDET, Misiones, Junio 2007.

Llull Gilet, A. (2003): Contabilidad medioambiental y desarrollo sostenible en el sector turístico, Govern de Les Illes Balears, España.

Lozano, J. (1999): Ética y Empresa, Madrid, Ed. Trota.

Organización Mundial del Turismo (1999): "Guía para Administradores locales: Desarrollo Turístico Sostenible”, Madrid, OMT.

Rozo, E. (2002): "El turismo sustentable como promotor del desarrollo de las comunidades locales" en Meyer Krumholz, D. (coord.) "Turismo y Desarrollo Sostenible”. Universidad Externado de Colombia, pp: 125-140.

Selltiz, C; Jahoda, M; Deutsch, M y Cook, S.W. (1976) en Schlüter, R. "Investigación en Turismo y Hotelería", CIET. 
Schlüter, R. (2005): Investigación en Turismo y Hotelería, Buenos Aires, CIET.

Vargas Martínez, E. (2007): “Gestión Ambiental y responsabilidad social empresarial (RSE) en el sector turístico de Cancún, Quintana Roo (México)". Protocolo Doctoral. UNAEM, México.

Winger, D. (1996) Cap. en "Naturaleza y (o) Lenguaje", en Daniel Winger (compilador) Naturaleza y(o) Lenguaje, UNR Editora.

Winger, D. (2008): Turismo y cultura en la posmodernidad. Dificultades y nuevos rumbos desde una lectura de la ética aplicada, ponencia presentada en el X Congreso Nacional y IV Internacional de Investigación Turística, a celebrarse del 26 al 28 de noviembre en la Ciudad de Toluca (México).

WTTC (Consejo Mundial de Viajes y Turismo) (2002): Working Paper. 